\title{
Retrospektive Bestimmung der elektromagnetischen Exposition durch analoge Rundfunksender im Rahmen von KiSS
}

\author{
J. Philipp ${ }^{1}$, H. Merzenich ${ }^{2}$, H. Brüggemeyer ${ }^{3}$, S. Schmiedel ${ }^{2}$, M. Blettner ${ }^{2}$, and J. Schüz ${ }^{4}$ \\ ${ }^{1}$ Südwestrundfunk, Stuttgart, Deutschland \\ ${ }^{2}$ Institut für Medizinische Biometrie, Epidemiologie und Informatik, Universität Mainz, Deutschland \\ ${ }^{3}$ Niedersächsischer Landesbetrieb für Wasserwirtschaft, Küsten- und Naturschutz, Hannover, Deutschland \\ ${ }^{4}$ Institute of Cancer Epidemiology, Danish Cancer Society, Copenhagen, Denmark
}

\begin{abstract}
Zusammenfassung. Im Rahmen einer epidemiologischen Fall-Kontroll-Studie zur Untersuchung eines möglichen $\mathrm{Zu}-$ sammenhangs zwischen der Häufigkeit kindlicher Leukämien und elektromagnetischer Strahlung (,KiSS“ - Kindliche Leukämien und Expositionen in der Umgebung von hochfrequenten Sendestationen) soll die Exposition durch leistungsstarke analoge Rundfunksender retrospektiv (Zeitraum 1982-2003) quantifiziert werden. Die zu betrachtenden Sendernetze für AM-Hörfunk, FM-Hörfunk und analoges Fernsehen unterscheiden sich nicht nur hinsichtlich der Modulationsart und der von den Einzelsendern abgestrahlten Leistung, sondern auch in der Netzkonfiguration und den je nach Wellenbereich verschiedenen Strahlungseigenschaften der Sendeantennen. Damit sind bei diesen drei Rundfunkdiensten sowohl die absolute Größe als auch die räumliche Struktur der hervorgerufenen Exposition verschieden. Es wird dargelegt, wie die für die Prognose der Rundfunkversorgung verwendeten Rechenverfahren zur Modellierung der Feldstärke für die Modellierung der Exposition herangezogen und durch Kontrollmessungen validiert werden und wie trotz Wahrung der Vertraulichkeit der dabei unabdingbar zu verwendenden Senderbetriebsdaten eine unerwünschte Beeinflussung der Studienergebnisse durch die Senderbetreiber ausgeschlossen wird.
\end{abstract}

\section{Einleitung}

Während sich die Funktechnik zu einem allgegenwärtigen und nicht mehr wegzudenkenden Mittel der Massen- wie der Individualkommunikation entwickelt hat, sind in Teilen der Bevölkerung erhebliche Besorgnisse über vermutete schädliche Auswirkungen der ubiquitären Exposition gegenüber elektromagnetischen Feldern auf die Gesundheit entstanden.

Correspondence to: J. Philipp

(johannes.philipp@swr.de)
Die Gesellschaft erwartet eine wissenschaftliche Objektivierung dieser Vermutungen durch Veri- oder Falsifikation. Wegen der Unmöglichkeit einer absoluten Falsifikation kann letzte Gewissheit nur im Fall einer Verifikation eintreten. Sollten die Vermutungen unbegründet sein, würde sich dies lediglich in einer Kette gescheiterter Verifikationsversuche niederschlagen können. Zwar würde jene von der Wissenschaft schließlich pragmatisch als bestmöglicher Falsifikationsersatz akzeptiert, zumal sich die potentiell noch bestehenden Risiken durch solche Untersuchungen immer mehr eingrenzen lassen. Es bleibt jedoch fraglich, ob damit alle Bedenken unter den Besorgten der Bevölkerung ausgeräumt werden können.

In der Literatur sind zahlreiche Einzeluntersuchungen an Tieren und in Zellkulturen dokumentiert, welche die Effekte schwacher elektromagnetischer Felder untersuchen. Insgesamt sind die Ergebnisse jedoch uneinheitlich und widersprüchlich. Bislang konnten bei Einwirkung von hochfrequenten elektromagnetischen Feldern bei Feldstärken unterhalb der gesetzlichen Grenzwerte (z.B. der 26. Verordnung zur Durchführung des Bundes-Immissionsschutzgesetzes) in wissenschaftlichen Studien keine krankheitsrelevanten Effekte nachgewiesen werden. Ebenso wenig ist es bisher gelungen, ein kausales Wirkungsmodell für eine Schädigung biologischer Systeme durch elektromagnetische Felder unterhalb der gesetzlichen Grenzwerte zu formulieren. Daher ist das Mittel der epidemiologischen Untersuchung ein wichtiges Instrument zur Identifizierung des Risikopotentials auf Bevölkerungsebene, das bei hinreichend großen Studienpopulationen recht gut eingegrenzt werden kann (vgl. Schüz, 2006).

Vor der Einführung der Mobilfunktechnik wurde eine hochfrequente Exposition der Allgemeinbevölkerung vornehmlich durch die Sender des Rundfunks verursacht. Ihre oft hohen Strahlungsleistungen und die Tatsache, dass sich der Betriebszustand gerade der Hochleistungssender über Jahrzehnte hinweg nur wenig geändert hat, lässt

Published by Copernicus Publications on behalf of the URSI Landesausschuss in der Bundesrepublik Deutschland e.V. 
epidemiologische Studien zu einer Assoziation zwischen beispielsweise Krebsinzidenz und hochfrequenter Exposition durch Rundfunksender besonders aussichtsreich erscheinen.

Die Aussagekraft bisher durchgeführter Studien zur Thematik (z.B. Dolk, 1997a, b) ist allerdings durch mehrere Umstände eingeschränkt. Zum einen erlaubt es ein verhältnismäßig geringer Umfang der Untersuchungspopulationen nicht, kleine Risiken mit ausreichender statistischer Sicherheit aufzudecken. Zum anderen wurde als Vergleichsmaß der Expositionsstärke stets ein geometrischer Abstand zur betrachteten Sendeanlage herangezogen. Feldstärke und Abstand sind jedoch insbesondere in der Nahumgebung von VHF- und UHF-Hochleistungssendern gewöhnlich nicht einfach korreliert. Vielmehr weist die Feldstärke dort wegen der vertikalen Bündelung der Sendeantennen lokale Maxima und Minima auf, worauf in (Cherry, 2001) zu Recht hingewiesen wurde. Die wesentlichste Einschränkung schließlich besteht darin, dass in allen bisherigen Studien keine individuelle Schätzung der Exposition erfolgt ist. Mit der Fall-KontrollStudie „KiSS“ (= Kindliche Leukämien und Expositionen in der Umgebung von hochfrequenten Sendestationen) wird versucht, diese genannten Beschränkungen zu vermeiden.

\section{Charakteristika und Modellierung der Exposition}

Die allgemeine Motivation und Methodik der KiSS-Studie sind an anderem Ort beschrieben (Brüggemeyer, 2007). Die spezielle Modellierung der Exposition wird durch Berechnung der Feldstärke am Wohnort der Fälle und Kontrollen bewerkstelligt. Messungen der Feldstärke wären zwar prinzipiell vorzuziehen. Angesichts der geplanten Studienpopulation von mehreren tausend Personen erscheint eine entsprechende Messkampagne jedoch undurchführbar. Außerdem sind aufgrund der jüngst begonnen Digitalisierung der Fernsehverbreitung viele der analogen Sender, die im für die Studie betrachteten Expositionszeitraum (1982-2003) maßgeblich zur Exposition beigetragen haben, schon seit einiger Zeit nicht mehr in Betrieb, so dass von aktuellen Messungen nicht mehr auf die frühere Exposition rückgeschlossen werden kann. Auch bei den AM-Sendern ist dies in der Regel nicht möglich. Ihre Betriebszustände haben sich in den letzten zwei Jahrzehnten einerseits infolge der deutschen Wiedervereinigung und andererseits im Zuge der Anpassung an neues Immissionsschutzrecht erheblich geändert.

Mit der Wahl der Feldstärke als Expositionsmaß wird der oben geschilderten Tatsache Rechnung getragen, dass der Abstand nur ein unzureichendes proxy für die Exposition darstellt. Außerdem bedingt die für die statistische Nachweiskraft erforderliche Größe der Studienpopulation ein entsprechend großräumiges Untersuchungsgebiet. In diesem kann die Exposition gewöhnlich nicht mehr auf einen einzigen dominanten Sender zurückgeführt werden. Vielmehr ist der gleichzeitige Einfluss mehrerer Sender zu berücksichtigen.
Dies wäre mit einem Abstandsmaß kaum noch sinnvoll zu erreichen (vgl. Abb. 1, Karte der Rundfunksendeanlagen im Großraum Stuttgart).

Die verschiedenen zur Exposition beitragenden Sender arbeiten mit unterschiedlichen Modulationsverfahren. Während die FM-Sender (UKW; Band II) ein Signal konstanter Leistung und Hüllkurve ausstrahlen, schwankt die Hüllkurve der AM-Signale (LW, MW, KW) mit der niederfrequenten Momentanspannung der übertragenen Audioinformation (leider können nur Gebiete ohne nennenswerte Exposition durch KW-Sender in der Studie berücksichtigt werden, da die tages- und jahreszeitlich stets wechselnden Betriebszustände der KW-Sendeanlagen nicht mit ausreichender Sicherheit rekonstruiert werden können). Die Strahlung der Sender des analogen Fernsehens (ATV; Band I, III, IV/V) kann als ,gepulst“ bezeichnet werden, da die mit einer Frequenz von $50 \mathrm{~s}^{-1}$ übertragenen Bild- und die (bei Bildern mit 625 Zeilen) mit $15625 \mathrm{~s}^{-1}$ übertragenen Zeilensynchronimpulse durch kurzzeitige Auftastung des Senders auf Maximalamplitude realisiert werden. Eine Untersuchung der immer wieder postulierten speziellen Wirkung dieser Strahlung wäre erleichtert, wenn es ein Gebiet gäbe, in dem sie die Exposition dominiert. Leider gibt es kaum Sendetürme, an denen neben Fernsehsendern nicht gleichzeitig auch leistungsstarke UKW-Sender installiert sind. Im Rahmen von KiSS wird die Exposition immerhin nach Modulationsarten getrennt erfasst. Damit wird auch dem Umstand Rechnung getragen, dass die Exposition bei den verschiedenen Modulationsarten und in den verschiedenen Frequenzbereichen nicht auf einfache Weise einheitlich beschrieben werden kann. Erstens sind die zur Verfügung stehenden Rechenmodelle heterogen (s.u.). Zweitens weist das Verhältnis der außerhalb von Gebäuden herrschenden Feldstärke zu den eigentlich expositionsrelevanten, aber nicht direkt berechenbaren Werten innerhalb von Gebäuden je nach Frequenzbereich verschiedenes Verhalten auf. Die Unkenntnis der jeweiligen Dämpfungseigenschaften der Wohngebäude der Fälle und Kontrollen ist eine Ursache möglicher Missklassifikationen bei der Expositionsbestimmung.

Die Unterschiede des Verhältnisses von Außen- zu Innenfeldstärke werden durch Gegenüberstellung von orientierenden Messungen deutlich, die im Gebäude der Stuttgarter Vogelsangschule (VHF/UHF) und in zwei Gebäuden in der Nähe des SWR-Senders Mühlacker (MW) durchgeführt wurden. Abbildung 2 zeigt frequenzbereichsweise (Band II, Band III, Band IV/V) die Differenz der in den Innenräumen gemessenen elektrischen Summenfeldstärke zur außen in $9 \mathrm{~m}$ Höhe gemessenen elektrischen Summenfeldstärke einschließlich der Standardabweichungen außen und innen (3 Messpunkte außen, 15 Messpunkte innen, Erdgeschoss und 1. Stock). Der mittlere Unterschied von etwa $12 \mathrm{~dB}$ beim untersuchten Gebäude ist sowohl auf die Eindringdämpfung als auch auf die Höhenabhängigkeit der Außenfeldstärke zurückzuführen. Die magnetische Feldstärke verhält sich in den betrachteten Frequenzbereichen vergleichbar. - 


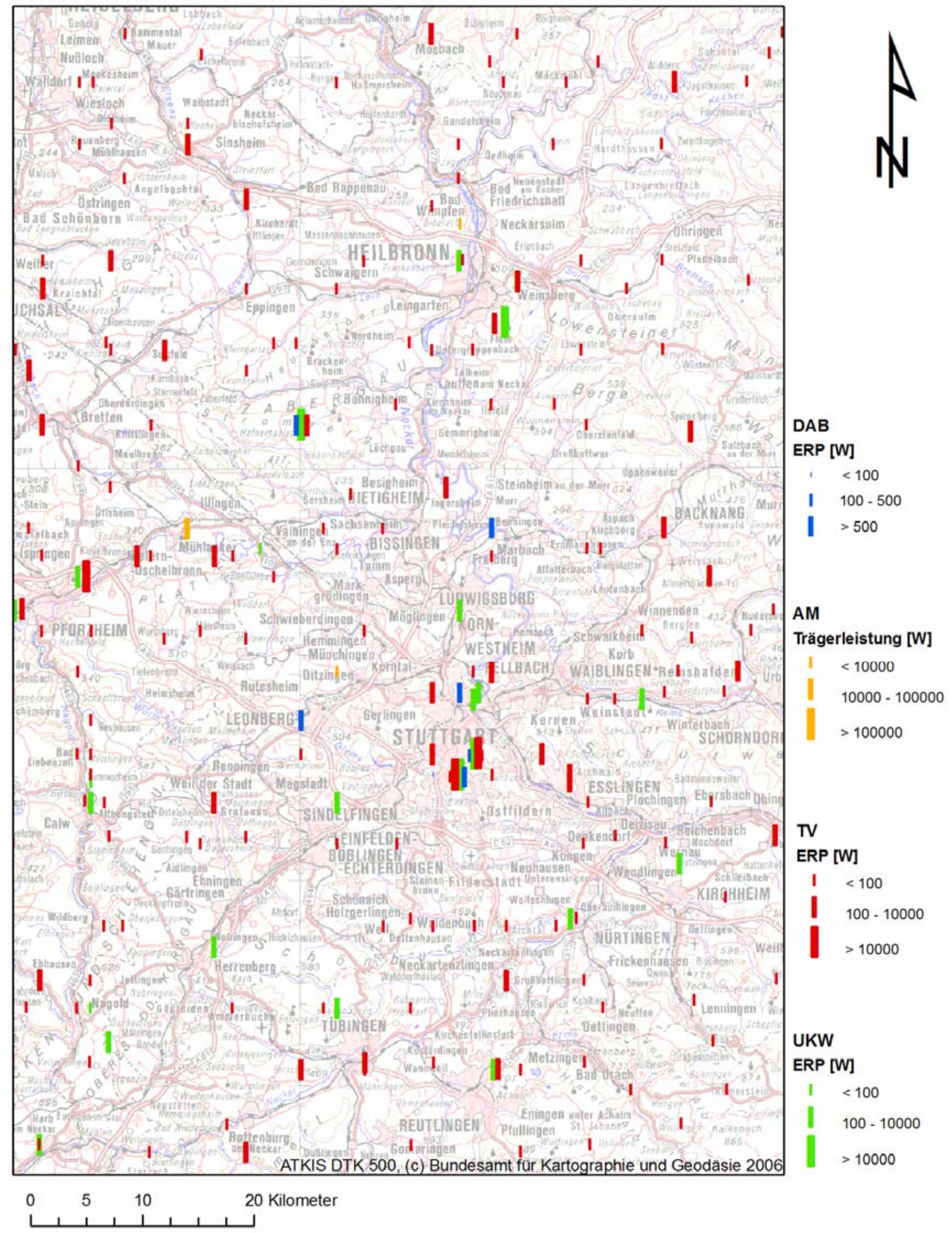

Abb. 1. Karte der Rundfunksendeanlagen im Großraum Stuttgart (Stand 2002). 


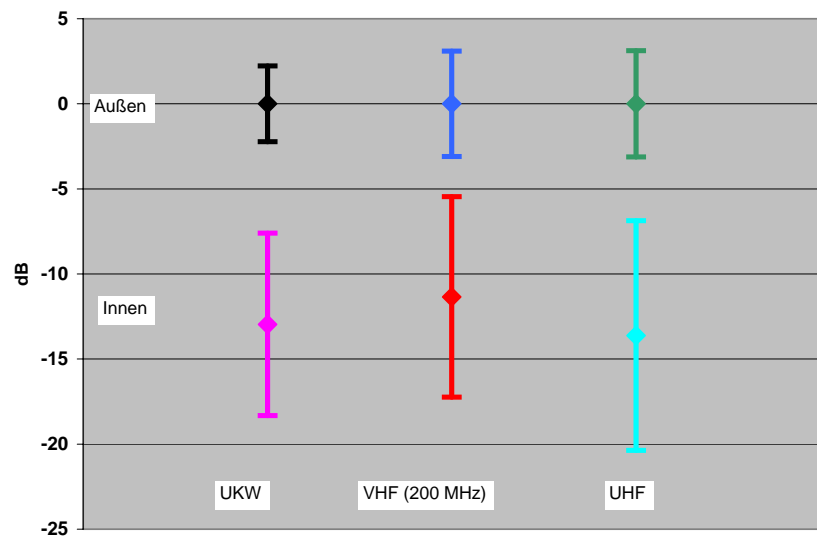

Abb. 2. Gebäudedämpfung am Beispiel der Vogelsangschule Stuttgart (E-Feld - Mittelwert des E-Feldes außen in $9 \mathrm{~m}$ Höhe über Grund).

Abbildung 3 zeigt die Feldstärkedifferenzen im MW-Bereich $(576 \mathrm{kHz})$ separat für die elektrische und die magnetische Feldstärke anhand zweier Gebäude, die hinsichtlich ihrer Dämpfungseigenschaften denkbare Extreme repräsentieren. Bei Gebäude 1 handelt es sich um einen Fachwerkbau auf gemauertem Erdgeschoss mit hölzerner Zwischendecke und Ziegeldach (Bücherei der Stadt Mühlacker), bei Gebäude 2 um einen Stahlbeton-Bungalow mit geerdetem Blechdach (Kantinengebäude auf dem Sendergelände des SWR). Bei Gebäude 1 zeigt sich eine erwartungsgemäß kleine Dämpfung bei der elektrischen Feldstärke, während die magnetische Komponente überhaupt keine Dämpfung aufweist, sondern sogar eine (nichtsignifikante) Erhöhung. Anders bei Gebäude 2: Hier erleidet die elektrische Feldstärke aufgrund der Abschirmung durch das Blechdach eine erhebliche Dämpfung, während die magnetische Feldstärke ähnlich abgeschwächt wird wie die elektrische beim Fachwerkhaus.

Schließlich ist zu bemerken, dass die Exposition der Allgemeinbevölkerung in den drei Frequenzbereichen Langbzw. Mittelwelle, VHF/Band II und VHF/Band III samt UHF/Band IV/V auf unterschiedlichen Skalen rangiert. Die minimal in Siedlungsgebieten anzutreffende Feldstärke ist jeweils durch die Auslegung des Sendernetzes bedingt, das ja eine Mindestanzahl von Programmen mit jeweils einer bestimmten Mindestfeldstärke im Versorgungsgebiet zur Verfügung stellen soll. Die maximal zu erwartenden Feldstärken hängen dagegen von der Konfiguration der einzelnen Sendeanlagen ab. Im MW-Bereich ist lokal gewöhnlich mindestens 1 Sender empfangbar. Dazu ist gemäß (ITU, 1975) eine Mindestfeldstärke von etwa $70 \mathrm{~dB}(\mu \mathrm{V} / \mathrm{m})$ erforderlich. Bei UKW-FM (Band II) sind gewöhnlich 4 Sender gleichzeitig empfangbar. Für jeden Sender ist nach (IRT 1982) eine Mindestfeldstärke von $54 \mathrm{~dB}(\mu \mathrm{V} / \mathrm{m})$ bei Stereoempfang in $10 \mathrm{~m}$ Höhe über Grund erforderlich, was sich bei 4 Programmen auf $60 \mathrm{~dB}(\mu \mathrm{V} / \mathrm{m})$ addiert. Diesem Wert entspricht mit dem üblichen Höhenabschlag von etwa

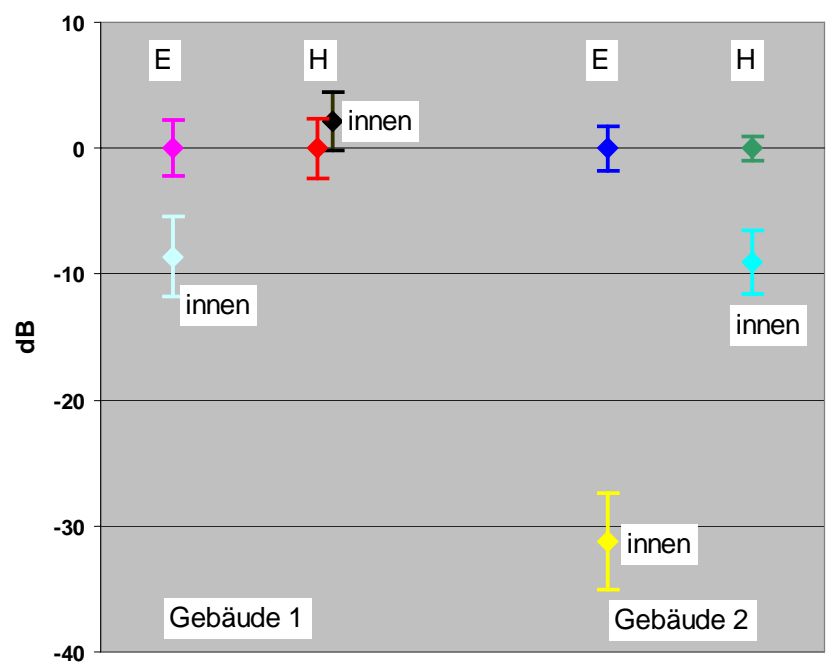

Abb. 3. Beispiele der Gebäudedämpfung im Mittelwellenbereich für E- und H-Feld, 1,5 m über Grund (normiert auf Mittelwert der Felder außen).

$10 \mathrm{~dB}$ ein Wert von $50 \mathrm{~dB}(\mu \mathrm{V} / \mathrm{m})$ in $1,5 \mathrm{~m}$ Höhe über Grund. Beim analogen Fernsehen waren in Deutschland lokal meist 3 Programme empfangbar. Mit der Mindestfeldstärke von etwa $70 \mathrm{~dB}(\mu \mathrm{V} / \mathrm{m})$ in $10 \mathrm{~m}$ Höhe über Grund für UHFProgramme und $58 \mathrm{~dB}(\mu \mathrm{V} / \mathrm{m})$ bei VHF (IRT, 1989) ergibt sich in der typischen Situation zweier UHF-Programme und eines VHF-Programms eine Mindest-Summenfeldstärke von $73 \mathrm{~dB}(\mu \mathrm{V} / \mathrm{m})$ in $10 \mathrm{~m}$ Höhe über Grund. Da sich Leistungspegel bei analogem Fernsehen gewöhnlich auf die während der Synchronimpulse ausgestrahlte maximale Leistung beziehen, die um $4 \mathrm{~dB}$ über der Durchschnittsleistung liegt, erhält man für die zu erwartende Mindest-Exposition mit dem Höhenabschlag von grob wieder $10 \mathrm{~dB}$ in $1,5 \mathrm{~m}$ Höhe über Grund einen Summen-Durchschnittspegel von etwa $60 \mathrm{~dB}(\mu \mathrm{V} / \mathrm{m})$. Die zu erwartenden maximal auftretenden Expositionsmaße können anhand typischer Senderkonfigurationen abgeschätzt werden. Nimmt man einen MWSender mit $100 \mathrm{~kW}$ Trägerleistung, $\lambda / 2$-Vertikalantenne und $500 \mathrm{~m}$ Abstand zur nächsten Wohnbebauung als Modell, so kommt man dort auf einen Feldstärkepegel von etwa $140 \mathrm{~dB}(\mu \mathrm{V} / \mathrm{m})$. Um einen Anhaltswert für typische FMHochleistungssender zu erhalten, wurde die Umgebung des Grundnetzsenders Stuttgart-Degerloch überschlägig vermessen. Die größte Summenfeldstärke in 1,5 m Höhe über Grund wurde in einer Entfernung von einigen $100 \mathrm{~m}$ vom Sendeturm beobachtet und beträgt etwa $130 \mathrm{~dB}(\mu \mathrm{V} / \mathrm{m})$.

Für typische Hochleistungssender des analogen Fernsehens in Band III und IV/V ist der maximale Durchschnittspegel der Feldstärke in 1,5 m Höhe über Grund aufgrund der schärferen vertikalen Bündelung der Sendeantennen um etwa $10 \mathrm{~dB}$ kleiner, beträgt also etwa $120 \mathrm{~dB}(\mu \mathrm{V} / \mathrm{m})$ in der Umgebung des Sendeturms. Die Ergebnisse der Betrachtungen sind in Tabelle 1 zusammengefasst. Um eine erste 


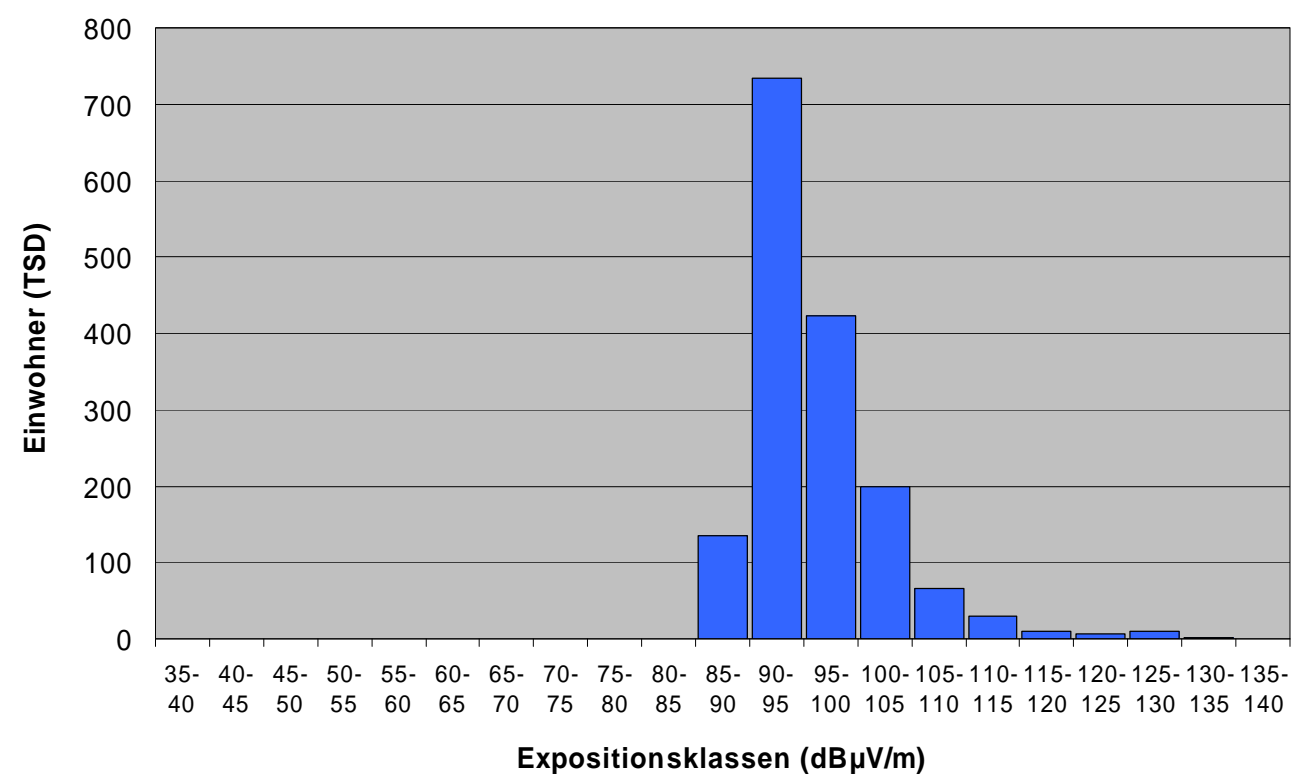

Abb. 4. Histogramm der Bevölkerungsexposition durch den MW-Sender Mühlacker im Umkreis von $30 \mathrm{~km}$.

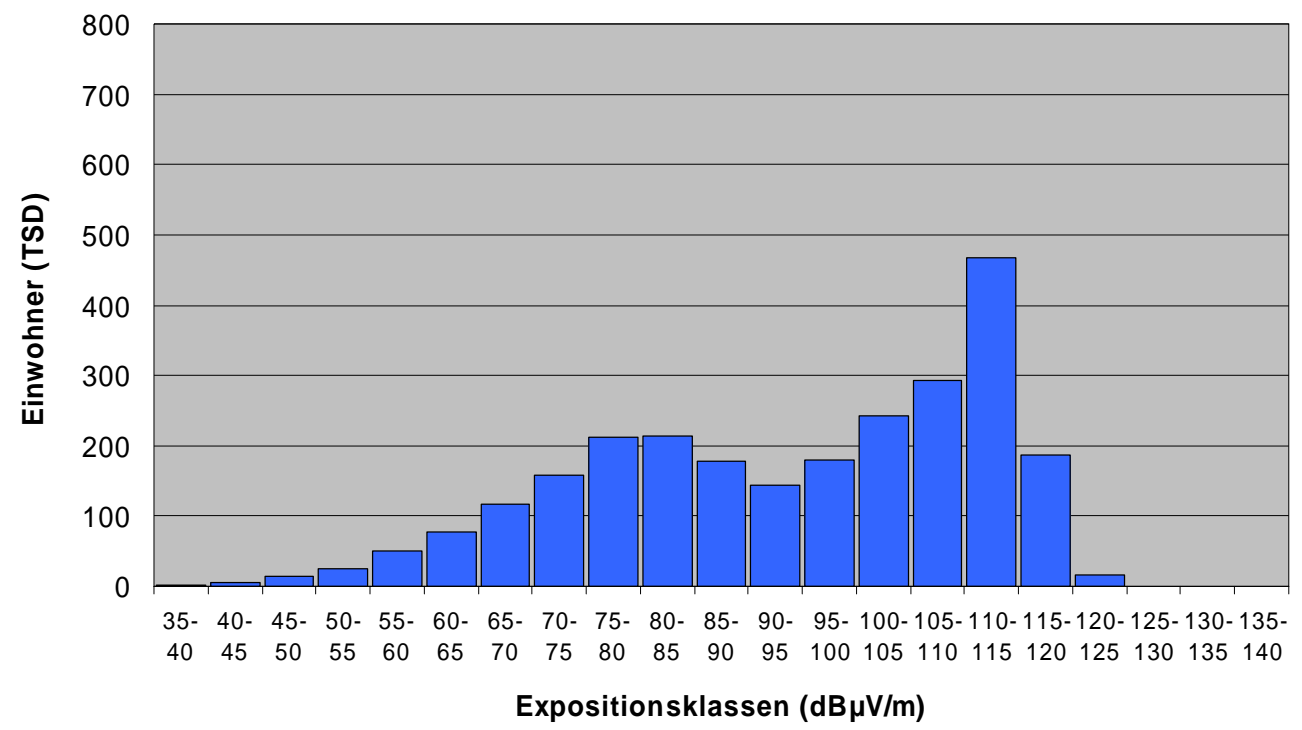

Abb. 5. Histogramm der Bevölkerungsexposition durch die UKW-Sender Stuttgart-Frauenkopf und Stuttgart-Degerloch im Umkreis von $30 \mathrm{~km}$.

Vorstellung von der Verteilung der Exposition innerhalb der abgeschätzten Grenzen zu erhalten, wurden beispielhaft eine beim SWR aus extrapolierten Daten der Volkszählung 1987 erstellte Datei der Flächenverteilung der Bevölkerung Baden-Württembergs mit berechneten und kumulierten Feldstärkewerten kombiniert. Abbildung 4 zeigt das so berechnete Histogramm der Bevölkerungsexposition durch den Mittelwellensender Mühlacker in $30 \mathrm{~km}$ Umkreis um den Sender. In Abb. 5 ist das Histogramm der berechneten Exposition mit Feldern der Stuttgarter UKW-FMSender in etwa $30 \mathrm{~km}$ Umkreis um die beiden Sendeanla- gen Stuttgart-Degerloch und Stuttgart-Frauenkopf wiedergegeben (Feldstärkewerte für $10 \mathrm{~m}$ über Grund). In beiden Fällen zeigt sich Kongruenz mit den pauschalen Expositionsgrenzen der Tabelle 1.

\section{Rechenmodelle für die Feldstärke}

Für die Feldstärkeberechnung bei Lang- und Mittelwelle (AM-Sender) wird das GRWAVE-Programm (ITU, 2005a) verwendet, das auf den Lösungen der 


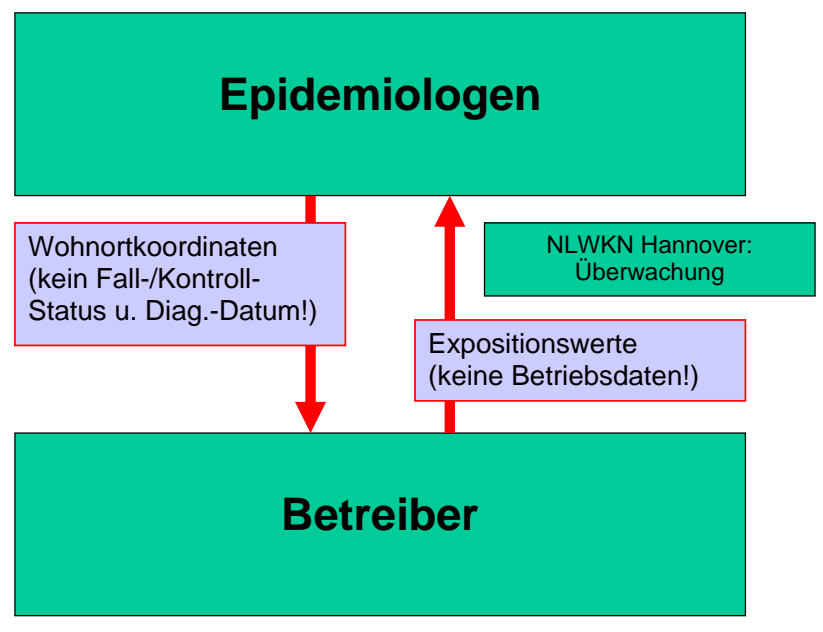

Abb. 6. Schema des Datenaustauschs zwischen epidemiologischer Forschungsgruppe und Sendeanlagenbetreibern.

van der Pol/Bremmer-Differentialgleichung für elektromagnetische Wellen über der gekrümmten Erde mit homogener Bodenleitfähigkeit beruht. Als Fehlerquelle und Ursache möglicher Missklassifikationen wirkt in erster Linie der Umstand, dass genaue Daten zur lokalen Bodenleitfähigkeit nicht vorliegen und dass die Topographie dabei nicht berücksichtigt werden kann. Die LW/MW-Rechnung liefert Werte für die Feldstärke am Boden.

Für den VHF- und UHF-Bereich wird ein Ansatz nach (Meeks, 1983) benutzt, der mehrfache Kantenbeugung an topographischen Hindernissen berücksichtigt. Berechnet wird der Medianwert der Feldstärke in einer Höhe von $10 \mathrm{~m}$ über Grund für Geländepixel in einer von der flächenhaften Auflösung der zugrundegelegten Topographiedaten abhängigen Größe. Diese beträgt typischerweise $100 * 100 \mathrm{~m}^{2}$; die Streuung des Feldstärkepegels im Pixel hängt stark von den Eigenschaften der Umgebung ab. Die in (ITU, 2005b) für die interessierenden Frequenzbereiche angegebenen Werte der Ortsstreuung liegen zwischen 5.3 und $9.5 \mathrm{~dB}$. Diese und die nicht modellierte Wirkung etwaiger Bodenreflexionen limitieren die Genauigkeit der Feldstärkeberechnung im VHFund UHF-Bereich.

Um die VHF- und UHF-Feldstärkewerte mit den LMWerten vergleichbar zu machen, müssten sie auf Werte in Bodennähe konvertiert werden. Dies ist nur mittels sehr grober Methoden wie z.B. Einrechnen eines pauschalen Höhenabschlags nach (ITU, 2004) möglich. Eine direkte Berechnung der Feldstärke in Bodennähe bei Frequenzen $>50 \mathrm{MHz}$ scheitert i.A. an der dann erforderlichen räumlichen Auflösung der digitalen Topo- und Morphographiemodellierung.
Tabelle 1. Abschätzung der bevölkerungsrelevanten Minimal- und Maximalwerte des Effektivwertes der Summenfeldstärke am Boden in den einzelnen Frequenzbereichen.

\begin{tabular}{lcc}
\hline & $\mathrm{E}_{\min } / \mathrm{dB}(\mu \mathrm{V} / \mathrm{m})$ & $\mathrm{E}_{\max } / \mathrm{dB}(\mu \mathrm{V} / \mathrm{m})$ \\
\hline Mittelwelle & 70 & 140 \\
UKW & 50 & 130 \\
analoges TV Bd III, Bd IV/V & 60 & 120 \\
\hline
\end{tabular}

\section{Datengrundlage}

Die Berechnung des Expositionsmaßes erfordert sowohl eine detaillierte Kenntnis der Betriebszustände aller relevanten Sendeanlagen während des Studienzeitraums sowie die Verfügbarkeit von digitalen Geländehöhenmodellen (DHM) für die Studiengebiete. Die verwendeten DHM (Flächenauflösung $100 * 100 \mathrm{~m}^{2}$ ) wurden aus photogrammetrischen Daten der Landesvermessungsämter abgeleitet, die den Rundfunkanstalten der ARD zur Verfügung stehen (vgl. Philipp, 1995). Hinsichtlich der Betriebsdaten der Sendeanlagen bot sich zunächst die Möglichkeit an, zwei bestehende umfangreiche Datenbanken der Bundesnetzagentur zu nutzen. Eine dient der Beschreibung und Realisierung der elektromagnetischen Sicherheit in der Umgebung einer jeden ortsfesten Sendeanlage gemäß der Verordnung über die Beschränkung elektromagnetischer Felder, die andere der Beschreibung und Begrenzung der Versorgungs- wie der gegenseitigen Störwirkungen der Sendeanlagen im Lichte der relevanten internationalen Frequenzpläne. Beide Dateien enthalten allerdings keine detaillierten Informationen über die wahren Betriebszustände der Sendeanlagen, sondern lediglich Toleranzschemata, die durch die jeweiligen Emissionen nicht überschritten werden dürfen. Es ist daher sinnvoll diese Daten nur dann zu verwenden, wenn keine weitergehende Information vorliegt. Glücklicherweise geben in den meisten Fällen das Betriebshandbuch der Anlagenbetreiber sowie bei diesen vorhandene Dokumente über die Strahlungsdiagramme der Antennen detailliertere Auskunft über die tatsächlichen historischen Betriebszustände, wenngleich im Zuge der Digitalisierung der Programmverbreitung diese Unterlagen obsolet werden und allzu leicht der Vernichtung anheim fallen.

Bei Betriebsdaten (Strahlungsleistung, horizontales und vertikales Strahlungsdiagramm der Sendeantenne, Montagehöhe der Antenne, Betriebszeiten, Modulationsparameter) handelt es sich größtenteils um Interna der Betreiber der Sendeanlagen. Kein Betreiber ist bereit, seine Betriebsdaten zu veröffentlichen. Hier schien eine ernsthafte Schwierigkeit bei der Konzeption der KiSS-Studie zu liegen. Wie können die für die Studie unabdingbaren Betreiberdaten verfügbar gemacht werden, ohne einerseits das Interesse der Betreiber an einer Vertraulichkeit der Daten zu verletzen und ohne 


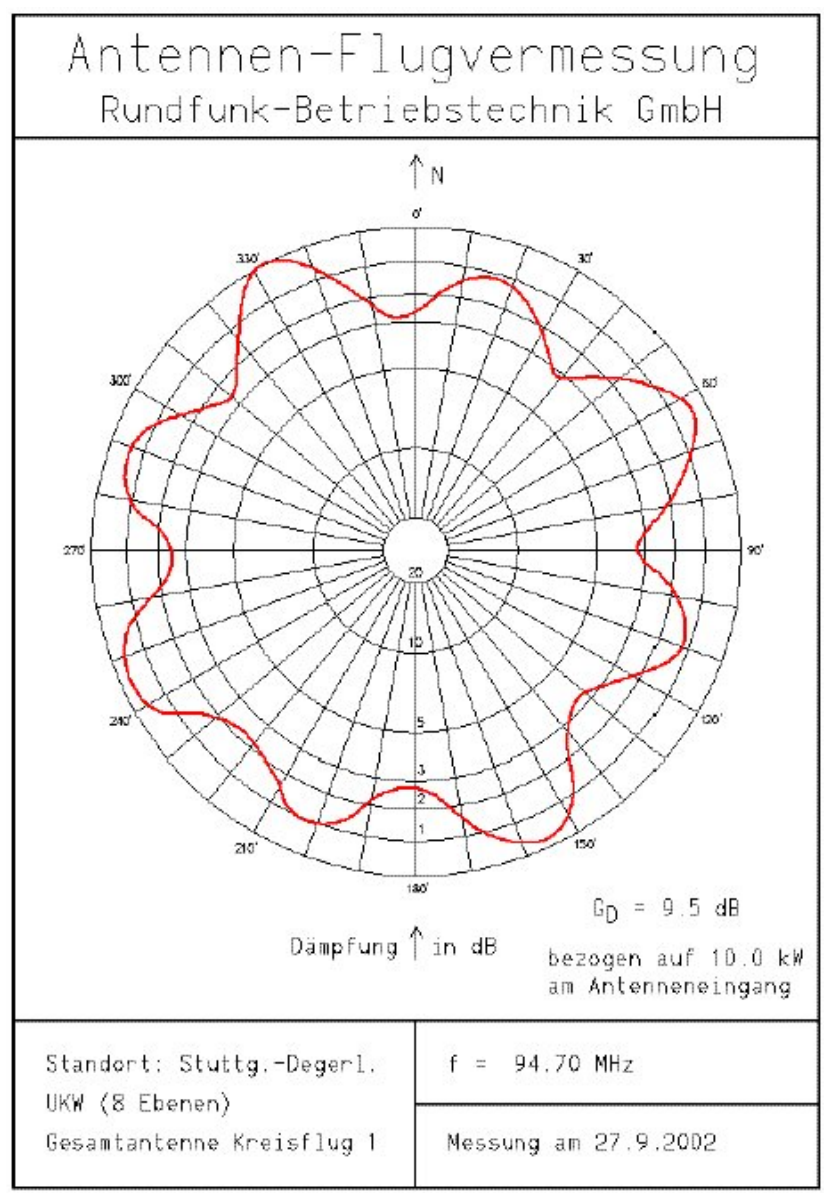

Abb. 7. Horizontaldiagramm der UKW-Antenne StuttgartDegerloch.

andererseits den Betreibern die Möglichkeit der Manipulation zu geben? Die gefundene praktikable Lösung ist in Abb. 6 schematisch dargestellt. Sie beruht darauf, dass die Betreiber, die der Nutzung ihrer Daten zugestimmt haben (die Rundfunkanstalten der ARD, Deutschlandradio, T-Systems International Media\&Broadcast GmbH, Fernmeldeverwaltung der Republik Tschechien), diese dem Südwestrundfunk (SWR) zur Auswertung im Rahmen der Studie zur Verfügung stellen. Der SWR wiederum ist verpflichtet, diese Daten einzig und allein für Zwecke der Studie zu nutzen und Dritten nicht zugänglich zu machen. Auf der Grundlage der Daten erstellt der SWR zeitliche Expositionsprofile für jedes Geländepixel der Untersuchungsgebiete. Das Epidemiologenteam der Universität Mainz ruft beim SWR für die Wohnortkoordinaten der ausgewählten Fall- und Kontrollpersonen diese zeitlichen Expositionsprofile ab. Dabei wird dem SWR weder der Fall-Kontroll-Status der jeweiligen Person noch das assoziierte Diagnosedatum mitgeteilt. Um die Möglichkeit von Manipulationen durch die Betreiber möglichst auszuschließen, werden Testsequenzen von Koordinaten in die Anfragen gemischt, deren betreiberseitiger Response von einer unabhängigen Institution (dem Niedersächsischen Lan-

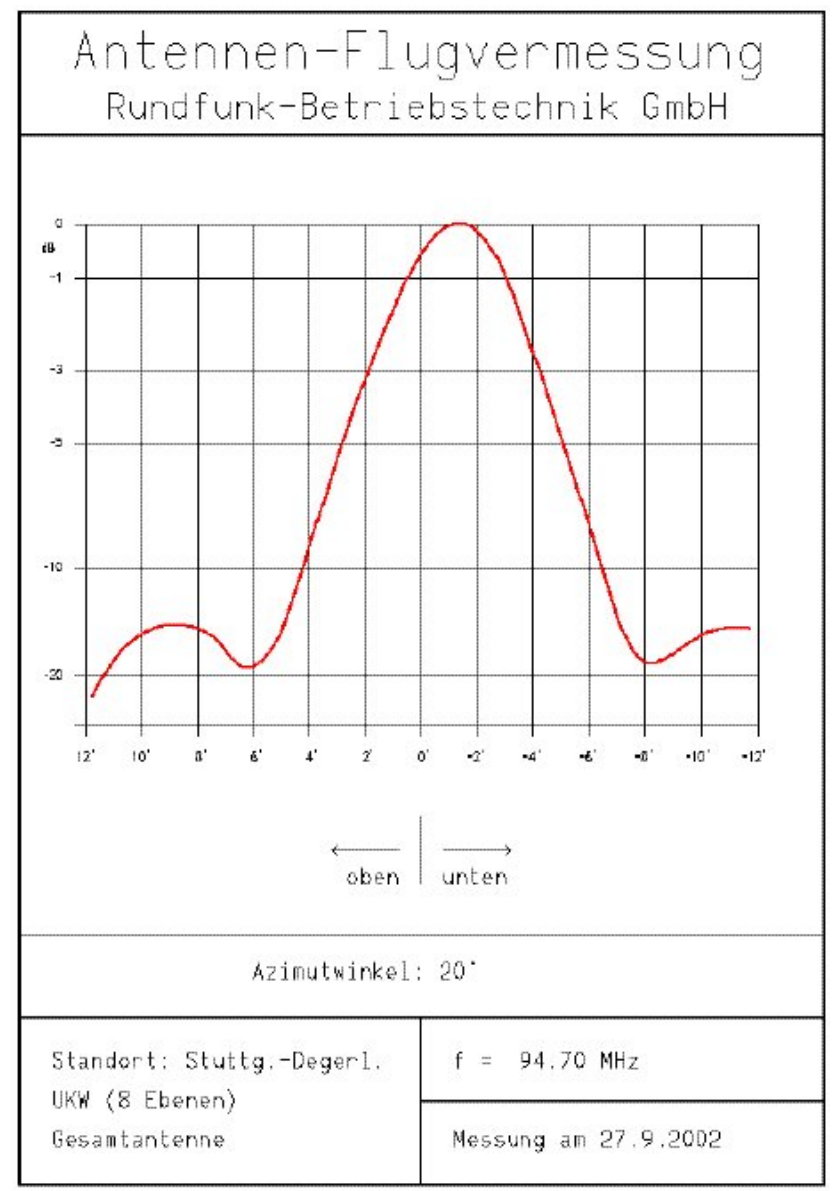

Abb. 8. Vertikaldiagramm der UKW-Antenne Stuttgart-Degerloch.

desbetrieb für Wasserwirtschaft, Küsten- und Naturschutz, Hannover) auf Plausibilität kontrolliert wird. Zudem werden die verwendeten Senderbetriebsdaten für spätere Überprüfungen archiviert.

Die für die flächenhafte Berechnung der Feldstärke benutzten Programme sind als Werkzeuge für eine Modellierung der Rundfunkversorgung entwickelt worden. Da in Sendernähe gewöhnlich ausreichende Feldstärken herrschen und Versorgungsprobleme nur ausnahmsweise auftreten, sind die eingesetzten Rechenverfahren nicht für eine genauere Modellierung das Nahbereichs um Sendeanlagen optimiert. Insbesondere ist es üblich, bei UKW- und TVVersorgungsprognosen das Vertikaldiagramm der Sendeantennen nicht zu berücksichtigen. Damit würde jedoch die im Nahbereich auftretende Exposition um eine Größenordnung überschätzt. Deshalb wurde das beim SWR implementierte Meeks-Verfahren so erweitert, dass Vertikaldiagramme eingerechnet werden können. Bei Hochleistungssendeanlagen liegen häufig die Ergebnisse von Flugvermessungen der Antennen vor. Neben dem vollständigen horizontalen Strahlungsdiagramm (vgl. Abb. 7) lassen sich daraus zumindest für einen gewissen Bereich des Vertikalwinkels 


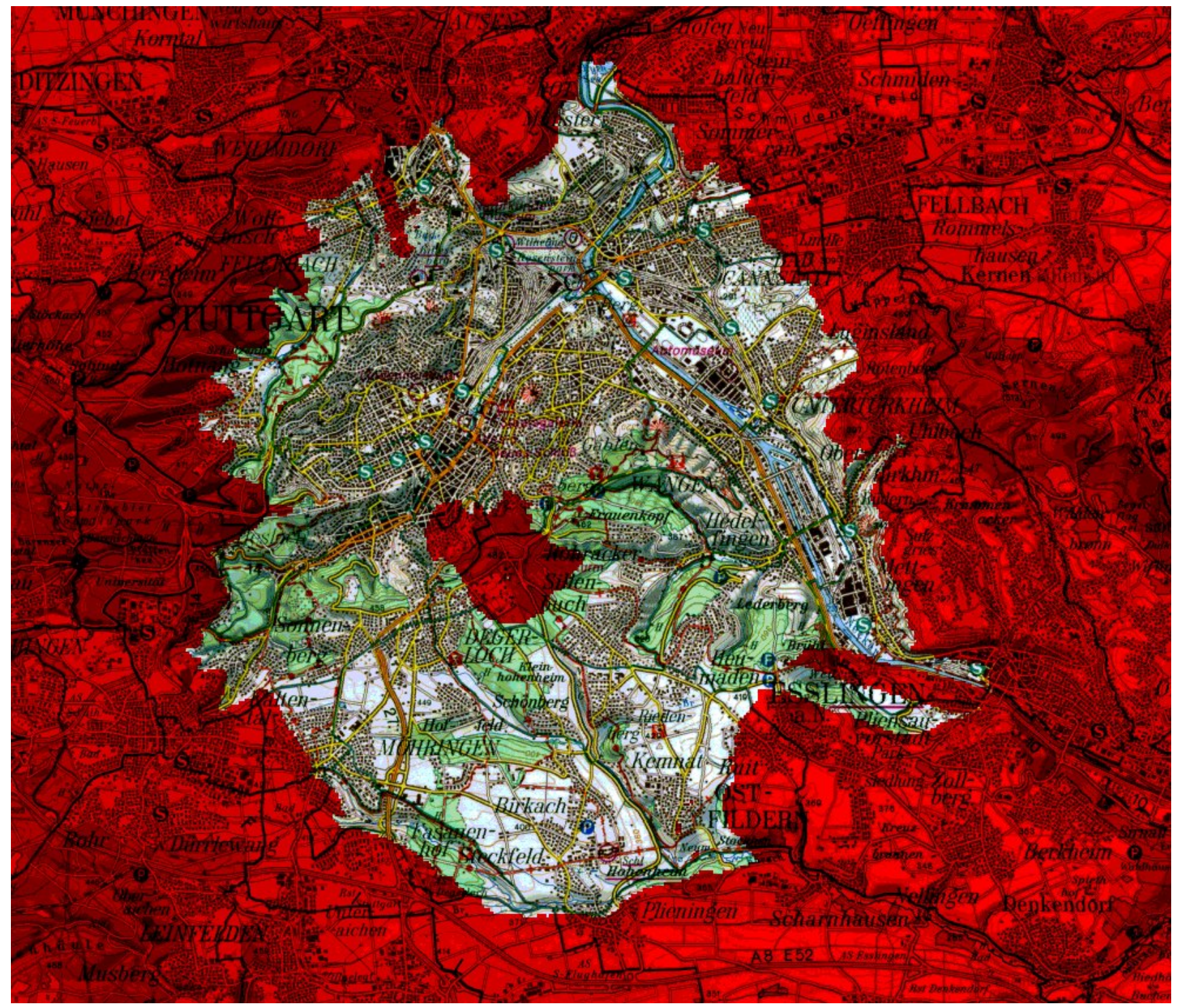

Abb. 9. Auswirkung des vertikalen Strahlungsdiagramms der UKW-Antenne Stuttgart-Degerloch. Äußeres rot gefärbtes Gebiet: Bereich des Hauptstrahls. Transparent dargestelltes Gebiet: Elevationswinkel zw. $-3^{\circ}$ und $-12^{\circ}$, abgeschwächte Exposition wegen vertikaler Bündelung. Inneres rot gefärbtes Gebiet: Elevationswinkel $<-12^{\circ}$, Extrapolationsbereich. Grundlage: Topographische Karte 1:100 000 Baden-Württemberg, (C) Landesvermessungsamt Baden-Württemberg (https://www.lv-bw.de), vom 25. Mai 2007, Az.: 2851.2-D/5884.

die Strahlungseigenschaften ablesen. Abbildung 8 zeigt das gemessene vertikale Strahlungsdiagramm einer während des Studienzeitraums vorhandenen UKW-Antenne des Senders Stuttgart-Degerloch. Hier liegen Daten bis zu einem Vertikalwinkel von $-12^{\circ}$ vor. Bei einer in einer Höhe von $200 \mathrm{~m}$ über ebenem Gelände montierten Antenne entspricht der nicht erfasste Winkelbereich von $-12^{\circ}$ bis $-90^{\circ}$ einem kreisförmigen Gebiet von etwa $1 \mathrm{~km}$ Radius, für den keine genaue Feldstärkeberechnung durchgeführt werden kann. Bei natürlicher Topographie ergeben sich andere Formen dieses Gebiets, wie sie z.B. in Abb. 9 für den Sender StuttgartDegerloch dargestellt sind. Um für solche Gebiete dennoch eine Expositionsberechnung vornehmen zu können, werden die gemessenen vertikalen Strahlungsdiagramme für kleinere Vertikalwinkel durch Extrapolationen ergänzt.

Bei der Modellierung der Exposition durch MW-Sender stellte sich das Problem, dass Unterlagen über horizontale Strahlungsdiagramme, welche den Betriebszustand der jeweiligen Anlage befriedigend widerspiegeln, nicht in allen Fällen existieren. Bei solchen Anlagen wurde die Antennenkonfiguration mit MININEC modelliert und daraus das Strahlungsdiagramm abgeleitet. 


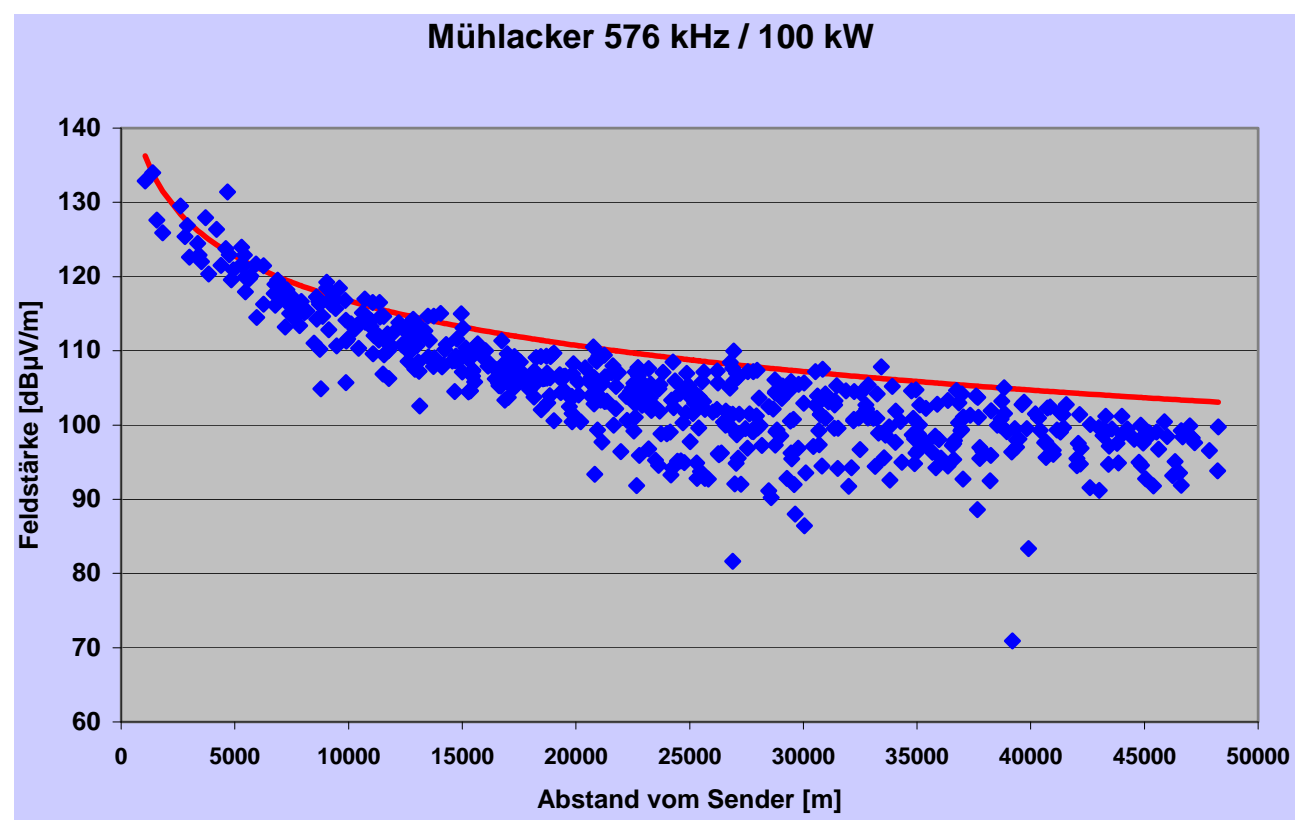

Abb. 10. Validierung der Feldstärkeberechnung für MW. Vergleich Rechnung (durchgezogene Kurve) - Messungen (Punkte).

\section{Validierung der Expositionsbestimmung}

Da sich das in der KiSS-Studie verwendete Expositionsmaß wesentlich auf die Berechnung der Feldstärke stützt, ist es unabdingbar, die verwendeten Rechenverfahren zu validieren. Dazu können die Ergebnisse der Rastermessungen (frequenzabhängige Feldstärke in $1,5 \mathrm{~m}$ Höhe über Grund) herangezogen werden, die im Auftrag der Karlsruher Landesanstalt für Umweltschutz in verschiedenen Gebieten Baden-Württembergs in den Jahren 2002-2003 durchgeführt wurden (Bochtler, 2003). Abbildung 10 zeigt einen Vergleich zwischen Rechnung und Messung für den MW-Sender Mühlacker $576 \mathrm{kHz}$. Dazu wurden die Messwerte nach der Entfernung des jeweiligen Messpunkts vom Sender geordnet und anhand des berechneten von der Kreisform leicht abweichenden horizontalen Strahlungsdiagramms des Senders so korrigiert, dass sie einem exakt kreisförmigen Strahlungsdiagramm entsprechen. Diese Werte sind denen mit GRWAVE berechneten gegenübergestellt. Die Unterschiede zwischen Messung und Rechnung beruhen u.a. auf der Tatsache, dass für die Bodenleitfähigkeit als wesentlicher Einflussgröße pauschale Annahmen getroffen werden müssen, weil lokale Werte nicht bekannt sind und die Topographie nicht berücksichtigt werden kann.

Auch für einige weitere Teile des Studiengebietes gibt es ähnliche hinreichend dokumentierte historische Messungen der Feldstärke, die zur Validierung herangezogen werden. Zudem stehen Messwerte zur Verfügung, die beim SWR im Rahmen der ständigen messtechnischen Überprüfung der Rundfunkversorgung gewonnen wurden (UKW, Feldstärke in 9 m Höhe über Grund). Durch Vergleich von Rechnungen und Messungen kann insbesondere auch abgeschätzt werden, welchen Beitrag in der Studie nicht berücksichtigte weitere Quellen elektromagnetischer Strahlung zur Gesamtexposition liefern.

\section{Literatur}

Bochtler, U., Eidher, R., und Wuschek, M.: Großräumige Ermittlung von Funkwellen in Baden-Württemberg, Aschaffenburg, Stuttgart und Regensburg, 2003.

Brüggemeyer, H., Philipp, J., Merzenich, H., Schmiedel, S., Blettner, M., und Schüz, J.: Kindliche Leukämien und EMFExpositionen in der Umgebung hochfrequenter Sendestationen (KiSS) - Fragestellung und Studienbeschreibung, Adv. Radio Sci., 5, 2007.

Cherry, N.: Re: "Cancer incidence near radio and television transmitters in Great Britain, I. Sutton Coldfield transmitter, II. All high power transmitters"(Letter), Am. J. Epidemiol., 153, 204 205, 2001.

Dolk, H., Shaddick, G., Walls, P., Grundy, C., Thakrar, B., Kleinschmidt, I., and Elliott, P.: Cancer Incidence Near Radio and Television Transmitters in Great Britain, I. Sutton Coldfield Transmitter, Am. J. Epidemiol., 145, 1-9, 1997a.

Dolk, H., Elliot, P., Shaddick, G., Walls, P., and Thakrar, B.: Cancer Incidence Near Radio and Television Transmitters in Great Britain, II. All High Power Transmitters, Am. J. Epidemiol., 145, 10-17, 1997b.

IRT (Institut für Rundfunktechnik): Richtlinie für die Beurteilung der UKW-Hörfunkversorgung (Mono und Stereo) bei ARD und DBP, Nr. 5R4, München, 1982.

IRT (Institut für Rundfunktechnik): Richtlinie zur Beurteilung der Fernsehversorgung, Nr. 5R10, München, 1989. 
ITU (International Telecommunication Union): Final acts of the Regional Administrative LF/MF Broadcasting Conference (Regions 1 and 3), Genf, 1975.

ITU (International Telecommunication Union): Resolutions of the First Session of the Regional Radiocommunication Conference for planning of the digital terrestrial broadcasting service in parts of Regions 1 and 3, in the frequency bands $174-230 \mathrm{MHz}$ and 470-862 MHz, Genf, 2004.

ITU (International Telecommunication Union): Recommendation ITU-R P.368-8, Ground-wave propagation curves for frequencies between $10 \mathrm{kHz}$ and $30 \mathrm{MHz}$, Genf, 2005a.

ITU (International Telecommunication Union): Recommendation ITU-R P.1546-2, Method for point-to-area predictions for terrestrial services in the frequency range $30 \mathrm{MHz}$ to $3000 \mathrm{MHz}$, Genf, 2005b.
Meeks, M. L.: VHF Propagation over Hilly, Forested Terrain, IEEE Trans. Ant. Pr., 31, 483-489, 1983.

Philipp, J. E.: ATKIS-Daten in der Rundfunksendernetzplanung, in: Kophstahl, E. und Sellge, H. (Hrsg.), Das Geoinformationssystem ATKIS und seine Nutzung in Wirtschaft und Verwaltung, Hannover, 161-166, 1995.

Schüz, J., Jacobsen, R., Olsen, J. H., Boice Jr., J. D., McLaughlin, J. K., and Johansen, Ch.: Cellular Telephone Use and Cancer Risk: Update of a Nationwide Danish Cohort, J. Natl. Cancer Inst., 98, 1707-1713, 2006. 